\title{
ON THE STRUCTURE OF SETS OF UNIQUENESS
}

\author{
RUSSELL LYONS
}

(Communicated by Richard R. Goldberg)

ABstract. We show that every $U_{0}$-set is almost a $W$-set.

It may be expected that if a Borel set $E \subset \mathbf{T} \stackrel{\text { def }}{=} \mathbf{R} / \mathbf{Z}$ cannot carry any Borel measure $\mu$ whose Fourier-Stieltjes coefficients $\hat{\mu}(n) \stackrel{\text { def }}{=} \int_{\mathbf{T}} e^{-2 \pi i n t} d \mu(t)$ vanish at infinity, then the arithmetic of $E$ is partially responsible. We shall show that this is precisely the case.

Recall the following definitions (see [3]).

DEFINITION. A Borel measure $\mu$ on $\mathbf{T}$ is a Rajchman measure if $\lim _{|n| \rightarrow \infty} \hat{\mu}(n)=$ $0 ; R$ denotes the set of Rajchman measures. A set $E$ is a set of uniqueness in the wide sense, or $U_{0^{-}}$set, if $\mu E=0$ for all $\mu \in R$. A Borel set $E \subset \mathbf{T}$ is a $W$-set if there is some strictly increasing sequence of integers $\left\{n_{k}\right\}_{k=1}^{\infty}$ such that for all $x \in E$, $\left\{n_{k} x\right\}$ has a nonuniform asymptotic distribution $\nu_{x}$.

Let us say that a set $E$ is almost in a class $C$ if for every positive Borel measure $\mu$ carried by $E$, there is a set $F \in C$ such that $\mu(E \backslash F)=0$. In [3], we showed that $\mu \in R$ if and only if $\mu E=0$ for all $E \in W$. This immediately implies that every $U_{0}$-set is almost in $W_{\sigma}$, where $W_{\sigma}$ is the class of sets which are countable unions of $W$-sets. Indeed, given $E \in U_{0}$ and $\mu$ a positive measure carried by $E$, we have that $\sup _{G \in W_{\sigma}} \mu G$ is attained. Since $\left.\mu\right|_{F} \notin R$ for all Borel $F \subset E$ unless $\mu F=0$, it is easy to see that $\sup _{G \in W_{\sigma}} \mu G=\|\mu\|$, whence the claim follows. We shall prove here the following stronger result.

THEOREM. A Borel set $E$ is a $U_{0}$-set if and only if $E$ is almost a $W$-set.

Of course, one direction is trivial since every $W$-set is a $U_{0}$-set. In the other direction, we shall prove a still stronger result. Recall [3] that $E$ is a $W_{1}$-set if $E$ is a $W$-set corresponding to asymptotic distributions $\nu_{x}$ with $\hat{\nu}_{x}(1) \neq 0$ for $x \in E$. We shall show that $U_{0}$-sets are in fact almost $W_{1}$-sets. Furthermore, with the definitions extended as in [3], $U_{0}$-sets are almost $W_{1}$-sets in all LCA groups. For related results, see $[\mathbf{1}$ and $\mathbf{2}]$.

LEMMA. Let $\mu$ be a positive $\sigma$-finite measure. Suppose that $f$ and $g$ are measurable functions such that for every $x$, either $f(x) \neq 0$ or $g(x) \neq 0$. Then there exists a countable set $K \subset] 0, \infty[$ such that if $\alpha \in] 0, \infty[\backslash K$, then $f(x)+\alpha g(x) \neq 0$ for $\mu$-a.e. $x$.

PROOF. Let $G_{\alpha}=\{x: f(x)+\alpha g(x)=0\}$. Then $G_{\alpha} \cap G_{\beta}=\varnothing$ if $\alpha \neq \beta$, whence $K=\left\{\alpha>0: \mu G_{\alpha}>0\right\}$ is at most countable.

Received by the editors September 25, 1986.

1980 Mathematics Subject Classification (1985 Revision). Primary 42A63; Secondary 11K99.

Key words and phrases. Uniqueness, asymptotic distribution modulo 1.

Research partially supported by an American Mathematical Society Research Fellowship. 
LEMMA. Let $\mu$ be a positive $\sigma$-finite measure. Suppose that $f_{n}$ are measurable functions bounded by 1 such that for every $x$, some $f_{n}(x)$ is not 0 . Then there exist $\alpha_{n}>0$ such that $\sum \alpha_{n}<\infty$ and $\sum \alpha_{n} f_{n}(x) \neq 0$ for $\mu$-a.e. $x$.

PROOF. It is easy to see that we may assume $\mu$ to be finite. Let $E_{n}=$ $\left\{x: f_{n}(x) \neq 0\right\}$. We define $\alpha_{n}$ inductively. Let $\alpha_{1}=1$. If $\alpha_{1}, \ldots, \alpha_{N}$ have been defined, then choose $\alpha_{N+1}<\alpha_{N} / 2$ such that $\sum_{n \leq N+1} \alpha_{n} f_{n}(x) \neq 0 \mu$-a.e. on $\bigcup_{n \leq N+1} E_{n}$ and also

$$
\mu\left(\left\{x \in \bigcup_{n \leq N} E_{n}:\left|\sum_{n \leq N} \alpha_{n} f_{n}(x)\right|<2 \alpha_{N+1}\right\}\right)<N^{-1} .
$$

Then if $\sum_{n \geq 1} \alpha_{n} f_{n}(x)=0$, we have for all $N$,

$$
\left|\sum_{n \leq N} \alpha_{n} f_{n}(x)\right|=\left|\sum_{n>N} \alpha_{n} f_{n}(x)\right| \leq \sum_{n>N}\left|\alpha_{n}\right|<2 \alpha_{N+1},
$$

whence

$$
\mu\left(\left\{x: \sum_{n \geq 1} \alpha_{n} f_{n}(x)=0\right\}\right)<N^{-1}+\mu\left(\left(\bigcup_{n \leq N} E_{n}\right)^{c}\right) .
$$

Since $N$ is arbitrary, it follows that $\sum_{n \geq 1} \alpha_{n} f_{n}(x) \neq 0 \mu$-a.e.

REMARK. It is not hard to show by using Fubini's theorem that, in fact, almost all choices of $\left\{\alpha_{n}\right\}$ satisfy the lemma, where, say, $\alpha_{n}$ is chosen independently and uniformly in $\left.] 0, n^{-2}\right]$. One may also show that except for a meager set of positive sequences in $l^{1}\left(\mathbf{Z}^{+}\right)$, any positive sequence $\left\{\alpha_{n}\right\}$ satisfies the lemma.

PROOF OF THE THEOREM. Let $E$ be a $U_{0}$-set and $\mu$ a positive Borel measure on $E$. Then by [3], there are $W_{1}$-sets $E_{m}$ such that $\mu\left(E \backslash \bigcup_{m \geq 1} E_{m}\right)=0$; such that if the sequence corresponding to $E_{m}$ is $\left\{n_{k, m}\right\}$, then $\left\{n_{k, m} x\right\}$ has an asymptotic distribution $\nu_{m, x} \mu$-a.e.; and such that for all subsequences $\left\{n_{k, m}^{\prime}\right\} \subset\left\{n_{k, m}\right\}$, $\left\{n_{k, m}^{\prime} x\right\}$ also has the asymptotic distribution $\nu_{m, x} \mu$-a.e. Note that $\hat{\nu}_{m, x}(1) \neq 0$ for $x \in E_{m}$. By the lemma, we may choose $\left\{\alpha_{m}\right\}$ such that $\alpha_{m}>0, \sum_{m \geq 1} \alpha_{m}=$ 1 , and $\sum_{m \geq 1} \alpha_{m} \hat{\nu}_{m, x}(1) \neq 0 \mu$-a.e. Let $\left\{n_{k_{i}, m_{i}}\right\}_{i=1}^{\infty}$ be any strictly increasing sequence such that for all $m$,

$$
\lim _{I \rightarrow \infty} \frac{1}{I} \operatorname{card}\left\{i \leq I: m_{i}=m\right\}=\alpha_{m} .
$$

Then it is easy to see by Weyl's criterion that $\left\{n_{k_{i}, m_{i}} x\right\}$ has the asymptotic distribution $\sum \alpha_{m} \nu_{m, x} \mu$-a.e. with $\left(\sum \alpha_{m} \nu_{m, x}\right)^{\wedge}(1) \neq 0 \mu$-a.e. That is, $F=\left\{x:\left\{n_{k_{i}, m_{i}} x\right\}\right.$ has an asymptotic distribution $\nu_{x}$ with $\left.\hat{\nu}_{x}(1) \neq 0\right\}$ is a $W_{1}$-set such that $\mu(E \backslash F)=$ 0 .

The extension to LCA groups is immediate, save for one subtlety. Namely, given a collection of sequences $\left\{\gamma_{k, m}\right\}_{k \geq 1} \subset \hat{G}(m \geq 1)$ with $\lim _{k \rightarrow \infty} \gamma_{k, m}=\infty$, we must be able to mix subsequences of them (in appropriate proportions) so as to obtain a sequence still tending to $\infty$. This is achieved by an easy adaptation of the proof of Theorem 14 in [3].

ACKNowledgment. I am grateful to Professor Yitzhak Katznelson for a simplifying argument and useful discussion. 


\section{BIBLIOGRAPHY}

1 Jean-Pierre Kahane and Raphaël Salem, Distribution modulo 1 and sets of uniqueness, Bull. Amer. Math. Soc. 70 (1964), 259-261.

2 Russell Lyons, Characterizations of measures whose Fourier-Stieltjes transforms vanish at infinity, Bull. Amer. Math. Soc. (N.S.) 10 (1984), 93-96.

3 , Fourier-Stieltjes coefficients and asymptotic distribution modulo 1, Ann. of Math. (2) 122 (1985), 155-170.

Department of Mathematics, Stanford University, Stanford, California 94305 